\title{
Óleos essenciais e tratamento térmico no controle pós-colheita de bolor verde em laranja
}

\author{
Eliane Aparecida Benato ${ }^{1}$; Thatyane Cristina Belletti²; Daniel Terao ${ }^{3}$; Daniel Andrade de Siqueira Franco ${ }^{1}$
}

${ }^{1}$ Centro Experimental Central do Instituto Biológico, Alameda dos Vidoeiros, 1097, Campinas, SP, 13101-680, ebenato@biologico.sp.gov. br; franco@biologico.sp.gov.br. ${ }^{2}$ Bolsista PIBIC/CNPq, graduanda Ciências Biológicas - PUC-Campinas, SP, thatybelletti11@hotmail.com. ${ }^{3}$ EMBRAPA - Meio Ambiente, Rod. SP 340, km 127,5, Jaguariúna, SP, 13820-000, daniel.terao@embrapa.br.

Autor para correspondência: Eliane Aparecida Benato (ebenato@biologico.sp.gov.br)

Data de chegada: 08/03/2017. Aceito para publicação em: 27/04/2017.

$10.1590 / 0100-5405 / 175659$

\section{RESUMO}

Benato, E.A.; Belletti, T.C.; Terao, D.; Franco, D.A.S. Óleos essenciais e tratamento térmico no controle pós-colheita de bolor verde em laranja. Summa Phytopathologica, v.44, n.1, p.65-71, 2018.

O bolor verde é a principal doença de frutos cítricos pós-colheita. Produtos e processos alternativos para controle de doenças de plantas vêm sendo cada vez mais requeridos. O objetivo deste trabalho foi avaliar a atividade antifúngica de óleos essenciais sobre Penicillium digitatum em laranjas, isoladamente ou em combinação com tratamento térmico. Para tanto, um isolado do fungo foi submetido, in vitro, a diferentes concentrações dos óleos de canela, capim-limão e palmarosa, em meio de cultura BDA, sendo avaliada também a atividade antifúngica dos compostos voláteis dos óleos. Além disso, foi realizado um estudo do efeito dos óleos sobre laranjas inoculadas, de modo curativo e protetivo. Laranjas foram inoculadas com $10 \mathrm{uL}$ de suspensão de conídios $\left(10^{5}\right.$ conídios $\left.\mathrm{mL}^{-1}\right)$, em dois períodos de incubação ( $4 \mathrm{~h}$ antes ou $24 \mathrm{~h}$ após os tratamentos). Os tratamentos com óleos essenciais por aspersão foram: testemunha, canela, capim-limão e palmarosa, a 0,5 e $1,0 \mathrm{~g} \mathrm{~L}^{-1}$, acrescidos de Tween ${ }^{20}$. Outro teste com óleo de canela, para verificar a melhor dose, foi realizado com 0,$0 ; 0,12 ; 0,25 ; 0,5$ e $1,0 \mathrm{~g} \mathrm{~L}^{-1}$ e um blend de canela $\left(0,12 \mathrm{~g} \mathrm{~L}^{-1}\right)$ e capim-limão $\left(0,12 \mathrm{~g} \mathrm{~L}^{-1}\right)$. $\mathrm{O}$ armazenamento foi a $25^{\circ} \mathrm{C}$ e $80 \%$ de umidade relativa (UR) por até 6 dias. Posteriormente, efetuou-se um teste em laranjas inoculadas $4 \mathrm{~h}$ antes dos tratamentos: testemunha; canela $\left(0,12 \mathrm{~g} \mathrm{~L}^{-1}\right)$; termoterapia $\left(60{ }^{\circ} \mathrm{C}\right.$ por 20 s); termoterapia + óleo de canela; imazalil (1000 $\left.\mathrm{mg} \mathrm{L}^{-1}\right)$. Armazenaram-se os frutos a $10{ }^{\circ} \mathrm{C} / 85 \%$ UR por 6 dias mais 3 dias em condições ambiente. In vitro, o óleo de canela foi o mais fungitóxico para $P$. digitatum, inibindo totalmente o índice de crescimento micelial em concentrações superiores a $0,5 \mathrm{~g} \mathrm{~L}^{-1}$ por contato e, reduzindo significativamente pelos seus constituintes voláteis. Sobre laranjas inoculadas, o óleo de canela foi mais efetivo como curativo e, capim-limão como protetivo. No teste screening, a dose de 0,12 $\mathrm{g} \mathrm{L}^{-1}$ do óleo de canela mostrou melhor resultado frente às doses superiores e ao blend de canela mais capim-limão. A combinação termoterapia seguida de aspersão de óleo de canela reduziu significativamente o desenvolvimento do bolor verde (AACPD) nos frutos (40,5\%), quando armazenados sob refrigeração; entretanto, o fungicida imazalil proporcionou um controle mais efetivo (97\%) durante o armazenamento prolongado.

Palavras-chave: Penicillium digitatum, Cinnamomun zeylanicum, Cymbopogon citratus, Cymbopogon martinii, controle alternativo.

\section{ABSTRACT}

Benato, E.A.; Belletti, T.C.; Terao, D.; Franco, D.A.S. Essential oils and thermal treatment in the postharvest control of green mold in orange. Summa Phytopathologica, v.44, n.1, p.65-71, 2018.

Green mold is the primary postharvest disease of citrus fruits. Alternative products and processes for plant disease control have been increasingly required. The aim of this study was to evaluate the antifungal activity of essential oils on $P$. digitatum in oranges, alone or in combination with thermal treatment. Thus, an isolate of the fungus was subjected, in vitro, to different concentrations of oils of cinnamon, lemongrass and palmarose, in PDA culture medium; the antifungal activity of the volatile compounds of oils was also evaluated. Furthermore, a study was conducted to verify the effect of oils on inoculated oranges, in a protective and curative mode. Oranges were inoculated with $10 \mathrm{uL}$ conidial suspension $\left(10^{5}\right.$ conidia $\left.\mathrm{mL}^{-1}\right)$, two incubation periods ( $4 \mathrm{~h}$ before or $24 \mathrm{~h}$ after treatments). The treatments with essential oils that were sprayed were: control, cinnamon, lemongrass and palmarose, at 0.5 and $1.0 \mathrm{~g} \mathrm{~L}^{-1}$, plus Tween ${ }^{20}$. Another test with cinnamon oil, to determine the best dose, was conducted by using $0.0 ; 0.12 ; 0.25 ; 0.5$ and $1.0 \mathrm{~g} \mathrm{~L}^{-1}$ and a blend of cinnamon $\left(0.12 \mathrm{~g} \mathrm{~L}^{-1}\right)$ and lemongrass $(0.12$ $\mathrm{g} \mathrm{L}^{-1}$ ). Storage was at $25{ }^{\circ} \mathrm{C}$ and $80 \%$ relative humidity (RH) for up to 6 days. Subsequently, a test was conducted in oranges inoculated $4 \mathrm{~h}$ before treatments: control; cinamon $\left(0.12 \mathrm{~g} \mathrm{~L}^{-1}\right)$; thermotherapy $\left(60^{\circ} \mathrm{C}\right.$ for $\left.20 \mathrm{~s}\right)$; thermotherapy + cinnamon oil; and imazalil $\left(1000 \mathrm{mg} \mathrm{L}^{-1}\right)$. The fruits were stored at $10{ }^{\circ} \mathrm{C} / 85 \% \mathrm{RH}$ for 6 days plus 3 days at room conditions. In vitro, cinnamon oil was most fungitoxic for $P$. digitatum, totally inhibiting the mycelial growth index at concentrations higher than $0.5 \mathrm{~g} \mathrm{~L}^{-1}$ by contact and significantly reducing it by its volatile compounds. For inoculated oranges, cinnamon oil was more effective as curative and lemon grass as protective. In the screening test, the dose of $0.12 \mathrm{~g} \mathrm{~L}^{-1}$ cinnamon oil showed better result compared to higher doses and to the blend of cinnamon and lemon grass. The thermotherapy combination followed by spraying of cinnamon oil significantly reduced green mold development (AACPD) in fruits $(40.5 \%)$ stored under refrigeration; however, the fungicide imazalil provided a more effective control (97\%) during prolonged storage.

Keywords: Penicillium digitatum, Cinnamomun zeylanicum. Cymbopogon citratus, Cymbopogon martinii, alternative control. 
O bolor verde, causado por $P$. digitatum Sacc., é a principal doença em pós-colheita de frutos cítricos em todo o mundo. $\mathrm{O}$ fungo produz inúmeros esporos, que ficam na superfície de frutos infectados e são dispersos no campo, no galpão de embalagem, nas câmaras de resfriamento, nas embalagens e no transporte. Os sintomas iniciais aparecem como uma mancha mole, aquosa, ligeiramente descolorida, que na sequência, é recoberta pelo micélio branco e esporos de coloração verde-oliva são produzidos. A área de esporulação é circundada por um halo de micélio branco e outro de aspecto aquoso. Com o desenvolvimento da podridão, o fruto inteiro é tomado por uma massa de esporos, facilmente dispersos pelo movimento do ar. $\mathrm{O}$ bolor verde se desenvolve mais rapidamente a $24^{\circ} \mathrm{C}$ e mais lentamente acima de $30^{\circ} \mathrm{C}$ e abaixo de $10^{\circ} \mathrm{C}$. A podridão é completamente inibida a $1{ }^{\circ} \mathrm{C}(7,22)$.

Fungicidas são utilizados para controlar esta doença, no entanto, é crescente a restrição ao uso de produtos químicos, pois podem prejudicar a saúde dos consumidores e o meio ambiente, além de selecionar estirpes resistentes dos patógenos quando usados continuamente (10).

Processos físicos para tratamento de frutos, como a termoterapia, despertam muito interesse, por não deixarem resíduos tóxicos e serem de baixo impacto ao meio ambiente. O tratamento térmico de frutos cítricos orgânicos, a $56^{\circ} \mathrm{C}$ por $20 \mathrm{~s}$, com simultânea escovação, reduziu a incidência de $P$. digitatum em 50\% (16). Forner et al. (9) também observaram que o tratamento térmico de laranjas por imersão $\left(52{ }^{\circ} \mathrm{C} / 2\right.$ min) reduziu a ocorrência de bolor verde nos frutos.

Além disso, o emprego de produtos naturais, como óleos essenciais e extratos de plantas medicinais e aromáticas, vêm se mostrando como uma opção viável para o tratamento de frutos pós-colheita $(1,8,12,20)$. Rozwalka et al. (17) estudaram extratos, decoctos e óleos essenciais de plantas medicinais e aromáticas no controle do crescimento micelial in vitro de Glomerella cingulata (Stonem.) Spaulding \& Schrenk e C. gloeosporioides (Penz.) Sacc., isolados de goiaba. Os melhores resultados foram obtidos com óleo essencial de capim-limão (Cymbopogon citratus L.) e cravo-da-india (Syzigium aromaticum (Linn.) Merrill. \& Perry.).

Anaruma et al. (2) testaram o uso de 28 tipos diferentes de óleos essenciais contra C. gloeosporioides de maracujá amarelo (Passiflora edulis Sims f. flavicarpa Deg), sendo que o óleo de C. citratus mostrou o controle mais efetivo da antracnose nos frutos pós-colheita. Lorenzetti et al. (13), estudando a bioatividade de 12 óleos essenciais sobre Botrytis cinerea Pers. ex Fr. de morango, constataram que os óleos de capim-limão, canela (Cinnamomun zeylanicum Blume), palmarosa (Cymbopogon martinii (Roxb.) Will. Watson) e menta (Mentha piperita Linn.) mostraram-se como os mais promissores no controle deste patossistema.

Mais especificamente sobre $P$. digitatum, encontra-se o trabalho de Yahyazadeh et al. (23), onde verificaram que os óleos de tomilho (Thymus vulgaris L.) e cravo (Eugenia caryophyllata Thumberg) inibiram o crescimento micelial, entretanto, os óleos de erva-doce (Foeniculum vulgare Mill.) e sálvia (Salvia officinalis L.) não exerceram efeito fungitóxico. O óleo de tomilho mostrou alto efeito fungicida no controle de bolor verde em laranja 'Valência', em comparação com óleo de menta (6).

Perez-Afonso et al. (15) observaram a efetividade dos compostos carvacrol e tymol (constituintes de óleos de orégano e tomilho) na inibição de $P$. digitatum e P. italicum Wehmer, in vitro, bem como, na redução das podridões em limões quando tratados pós-colheita com os óleos incorporados à cera. Há também resultados positivos no controle de $P$. digitatum com óleos de capim-limão $(1,10)$; de canela $(3,14)$ e de palmarosa $(5)$.
A avaliação do efeito antimicrobiano in vitro e in vivo é interessante, haja visto o trabalho de Shao et al. (18), que constataram um efeito sinérgico da mistura de quitosana com óleo de canela no controle de $P$. digitatum in vitro, porém, quando aplicada em laranjas, a mistura não controlou o bolor verde.

O objetivo deste estudo foi avaliar o efeito fungitóxico de óleos essenciais sobre o patógeno $P$. digitatum, in vitro e sobre laranjas inoculadas, bem como, em combinação com tratamento térmico dos frutos.

\section{MATERIAL E MÉTODOS}

\section{Óleos essenciais e isolado do patógeno}

Foram empregados três óleos essenciais nos experimentos: 1) capim-limão (Cymbopogon citratus) - Laszlo ${ }^{\mathbb{B}}$;2) palmarosa (Cymbopogon martinii) - BioEssência ${ }^{\circledR}$, ambos da família Poaceae; 3) canela (Cinnamomun zeylanicum), família Myrtaceae - BioEssência ${ }^{\circledR}$. Os componentes majoritários são, respectivamente: citral, geraniol e eugenol $(11,13)$.

Utilizou-se um isolado de $P$. digitatum de laranja 'Pera', procedente de pomar de Engenheiro Coelho/SP, em 2014, cedido pela EMBRAPA- Meio Ambiente (L.02/14). O isolado foi preservado em tubo de ensaio com meio de cultura BDA (batatadextrose-ágar), acrescido de $2 \mathrm{~mL}$ de óleo mineral esterilizado. Antes de realizar os testes in vitro e in vivo, foi efetuado um teste de patogenicidade com o isolado.

Bioatividade de óleos essenciais no crescimento micelial de Penicillium digitatum

a) Atividade antifúngica dos óleos essenciais por contato

Os óleos essenciais foram incorporados ao meio de cultura BDA fundente $\left(50{ }^{\circ} \mathrm{C}\right)$, acrescido de Tween ${ }^{20}(0,05 \mathrm{~mL})$ e vertidos em placas de Petri $(90 \mathrm{~mm})$. As concentrações dos óleos testadas foram 0,$0 ; 0,12 ; 0,25 ; 0,50$ e $1,0 \mathrm{~g} \mathrm{~L}^{-1}$. Após $24 \mathrm{~h}$, no centro de cada placa de Petri, foi disposto um disco $(5 \mathrm{~mm})$ da borda da colônia do patógeno em crescimento há 7 dias, ficando as estruturas do fungo em contato com o meio de cultura. Em seguida, as placas foram acondicionadas em incubadora para BOD a $25^{\circ} \mathrm{C}$, com fotoperíodo de $12 \mathrm{~h}$, por 7 dias.

A avaliação foi feita pela medição do diâmetro da colônia do fungo (duas medidas ortogonais), diariamente, até que algum tratamento atingisse o diâmetro da placa. Os valores obtidos foram utilizados para calcular o índice de crescimento micelial $-\mathbf{I C M}=[\Sigma$ $(\mathbf{D}-\mathbf{D a})+(\ldots)] / \mathbf{N}$, onde: $\mathrm{D}=$ diâmetro médio atual; $\mathrm{Da}=$ diâmetro médio do dia anterior; $\mathrm{N}=$ número de dias após a repicagem. $\mathrm{O}$ delineamento experimental foi inteiramente casualizado, com cinco tratamentos por óleo essencial, com sete repetições e uma placa por parcela. Aplicou-se regressão polinomial sobre os resultados.

b) Atividade antifúngica de compostos voláteis dos óleos essenciais

Para avaliar o efeito dos compostos voláteis, uma alíquota (10 $\mu \mathrm{L}$ ) de cada óleo essencial foi depositada em um disco de papel-filtro esterilizado $(5 \mathrm{~mm})$ que foi colocado em um dos compartimentos da placa de Petri (com dois compartimentos), enquanto um disco (5 mm) do micélio do patógeno foi depositado no compartimento oposto sobre BDA, com o micélio voltado para cima. O espaço livre de cada placa de Petri foi de aproximadamente $58 \mathrm{~mL}$. Os tratamentos foram constituídos da testemunha (água destilada esterilizada) e três óleos essenciais. As placas foram seladas com fita adesiva e acondicionadas a $25^{\circ} \mathrm{C}$, com 
fotoperíodo de $12 \mathrm{~h}$ por 4 dias, sendo avaliadas, diariamente, medindose o diâmetro da colônia. O delineamento experimental foi inteiramente casualizado, com quatro tratamentos, sete repetições por tratamento, com uma placa por parcela. Com os dados obtidos calculou-se o ICM. Este teste foi repetido duas vezes.

\section{Bioatividade dos óleos essenciais em laranjas inoculadas}

a) Matéria-prima e inoculação

Laranjas, disponíveis na época dos ensaios - 'Valência' (nov./ dez.) e 'Pêra' (fev./mar./abr.), provenientes de pomares comerciais, recém-colhidas e em estádio de maturação adequado para consumo, foram transportadas para o laboratório, onde foram selecionadas e padronizadas. Preliminarmente, os frutos foram lavados com sabão neutro, sanitizados com hipoclorito de sódio (200 uL L-1 de cloro) por 10 min e enxaguados em água corrente e, após secagem com ventilador, submetidos aos tratamentos.

$\mathrm{O}$ isolado de $P$. digitatum foi cultivado em meio de cultura BDA, em incubadora BOD a $25^{\circ} \mathrm{C}$, com alternância de luz $(12 \mathrm{~h})$ por 7 dias. Para obtenção da suspensão de conídios, adicionou-se água destilada esterilizada nas placas de Petri com a cultura do fungo e, com auxílio de uma alça de Drigalsky, foi promovida a formação da suspensão dos conídios e micélio. A suspensão foi filtrada em gaze e ajustada para aproximadamente $10^{5}$ conídios $\mathrm{mL}^{-1}$, determinada pela contagem em hemocitômetro. Para melhor dispersão dos conídios na suspensão, adicionou-se uma gota de Tween ${ }^{20}$. Os frutos foram inoculados em um ponto da região equatorial, fazendo-se um microferimento (1-2 mm de profundidade), com auxílio de uma agulha com ponta em bisel, sobre o qual foram depositados $10 \mu \mathrm{L}$ da suspensão de conídios. Os frutos inoculados permaneceram em incubação a $25^{\circ} \mathrm{C}$ e alta umidade relativa.

b) Avaliação do efeito curativo dos óleos essenciais

Para avaliação do efeito curativo, foi realizada a inoculação de laranjas 'Valência' 4 horas antes da aplicação dos tratamentos com óleos. Os tratamentos foram: testemunha (água), canela, capimlimão e palmarosa $\left(0,5\right.$ e $\left.1,0 \mathrm{~g} \mathrm{~L}^{-1}\right)$. A solução de óleo essencial foi preparada com o acréscimo de Tween ${ }^{20}(0,05 \mathrm{~mL})$ mais água destilada. A aplicação nos frutos foi feita por aspersão. Após a secagem, os frutos foram acondicionados em caixas de papelão e envolvidos por sacos de polietileno de baixa densidade $(20 \mu \mathrm{m})$.

As laranjas foram armazenadas em incubadora BOD, a $25^{\circ} \mathrm{C} / 80 \%$ UR, pelo período de 4 dias. A avaliação do diâmetro da lesão nos frutos foi realizada diariamente, com paquímetro digital. Os diâmetros das lesões foram usados para calcular a área abaixo da curva de progresso da doença (AACPD), através da fórmula: AACPD $=\sum\left[\left(\mathbf{Y}_{\mathbf{i}+1}+\mathbf{Y}_{\mathbf{i}}\right) / 2\right]$ $\left[\mathbf{T}_{\mathrm{i}+1}-\mathbf{T}_{\mathrm{i}}\right]$; onde $\mathrm{Y}_{\mathrm{i}+1}=$ diâmetro da lesão no tempo $\mathrm{T}_{\mathrm{i}+1}$ e $\mathrm{Y}_{\mathrm{i}}=$ diâmetro da lesão no tempo $\mathrm{T}_{\mathrm{i}}$ (4). O delineamento experimental foi inteiramente casualizado, com sete tratamentos, constituídos de 10 repetições cada, com um fruto por parcela.

c) Avaliação do efeito protetivo dos óleos essenciais

Para avaliação do efeito protetivo, foi realizada a inoculação de laranjas 'Pera' 24 horas após a aplicação dos tratamentos com óleos, os mesmos descritos anteriormente. As laranjas foram armazenadas em incubadora BOD, a $25{ }^{\circ} \mathrm{C} / 80 \%$ UR, pelo período de 6 dias. A avaliação do desenvolvimento da podridão nos frutos foi feita diariamente, usando-se os diâmetros das lesões para calcular AACPD. $\mathrm{O}$ delineamento experimental foi inteiramente casualizado, com sete tratamentos, constituídos de 10 repetições cada, com um fruto por parcela. d) Avaliação de diferentes concentrações do óleo de canela

Com base nos resultados dos ensaios anteriores, selecionou-se o óleo essencial de canela como o mais promissor, assim foi realizado um teste screening para avaliar diferentes doses no controle do bolor verde em laranjas inoculadas.

Primeiramente, as laranjas 'Pêra' foram higienizadas e inoculadas e, após 4 horas, os frutos foram submetidos aos tratamentos. Aplicaram-se seis tratamentos: canela $\left(0,0 ; 0,12 ; 0,25 ; 0,5 ; 1,0 \mathrm{~g} . \mathrm{L}^{-1}\right)$ e canela $(0,12 \mathrm{~g}$. $\left.\mathrm{L}^{-1}\right)+$ capim-limão $\left(0,12 \mathrm{~g}\right.$. $\left.\mathrm{L}^{-1}\right)$. As soluções foram preparadas em água e acrescidas de Tween ${ }^{20}$. As laranjas foram armazenadas em incubadora $\mathrm{BOD}$, a $25^{\circ} \mathrm{C} / 80 \% \mathrm{UR}$, pelo período de 3 dias. A avaliação da podridão nos frutos foi realizada diariamente. $\mathrm{O}$ delineamento foi inteiramente casualizado, com seis tratamentos, constituídos de 10 repetições cada, com um fruto por parcela.

\section{Ensaio com óleo essencial e tratamento térmico}

Laranjas 'Pêra', provenientes de Engenheiro Coelho/SP, em estádio adequado para consumo, foram transportadas ao laboratório, onde passaram por seleção e higienização. Posteriormente, os frutos foram inoculados com $P$. digitatum. Após 4 horas de incubação, os frutos foram submetidos aos seguintes tratamentos: 1) testemunha (água); 2) canela $\left.\left(0,12 \mathrm{~g} \mathrm{~L}^{-1}\right) ; 3\right)$ tratamento térmico $\left(60^{\circ} \mathrm{C} / 20 \mathrm{~s}\right)$ por aspersão e escovação (equipamento disponível na EMBRAPA - Meio Ambiente); 4) tratamento térmico seguido de óleo de canela $(0,12 \mathrm{~g}$ $\mathrm{L}^{-1}$ ); 5) fungicida imazalil (Magnate $500 \mathrm{EC}\left(\right.$ ) $-1000 \mathrm{mg}$ i.a. $\mathrm{L}^{-1} \mathrm{em}$ imersão por 2 min. Nos tratamentos 1 a 4 , empregou-se Tween ${ }^{20}$ como espalhante adesivo e foram aplicados nos frutos por aspersão. As laranjas foram armazenadas a $10{ }^{\circ} \mathrm{C} / 85 \%$ UR pelo período de 6 dias mais 3 dias sob condições ambiente $\left(22^{\circ} \mathrm{C} / 70 \%\right.$ UR). A avaliação do diâmetro das lesões nos frutos foi feita aos 2 e 6 dias de armazenamento refrigerado e, diariamente, sob condições ambiente. Os valores foram usados para calcular AACPD. O delineamento experimental foi inteiramente casualizado, com cinco tratamentos, com seis repetições por tratamento, com seis frutos por parcela.

\section{Análise Estatística}

As médias dos resultados dos testes, in vitro e in vivo, foram submetidas a análise de variância e comparadas pelo teste de Tukey, a 5\% de probabilidade, usando o programa Estat 2.0. Quando necessário, os dados foram transformados em $\sqrt{x+0,5}$.

\section{RESULTADOS E DISCUSSÃO}

Nos resultados dos testes, in vitro, com a exposição de $P$. digitatum por contato a concentrações crescentes dos óleos essenciais (Figura 1), pode-se notar que o óleo de canela reduziu significativamente (Tukey, $\mathrm{p} \leq 0,05)$ a velocidade de crescimento do fungo (ICM), a 0,5 e $1,0 \mathrm{~g} \mathrm{~L}^{-1}$, sendo o modelo matemático polinomial de terceiro grau o que mais se ajustou aos resultados $\left(\mathrm{R}^{2}=0,96\right)$. Porém, em contato com os óleos de capim-limão e palmarosa, o fungo apresentou incremento significativo do ICM, respectivamente, a partir de 0,25 e $0,12 \mathrm{~g} \mathrm{~L}^{-1}$, ajustando-se aos modelos matemáticos quadrático $\left(\mathrm{R}^{2}=0,89\right)$ e de $3^{\circ}$ grau $\left(\mathrm{R}^{2}=0,96\right)$.

Quanto às propriedades fungitóxicas dos constituintes voláteis dos óleos essenciais, notou-se que o óleo de canela reduziu significativamente o ICM do fungo. O efeito do óleo de capimlimão mostrou redução nos valores de diâmetro da colônia, de modo significativo, apenas nos primeiros dias de incubação, mas não significativo para o ICM (Tabela 1). 


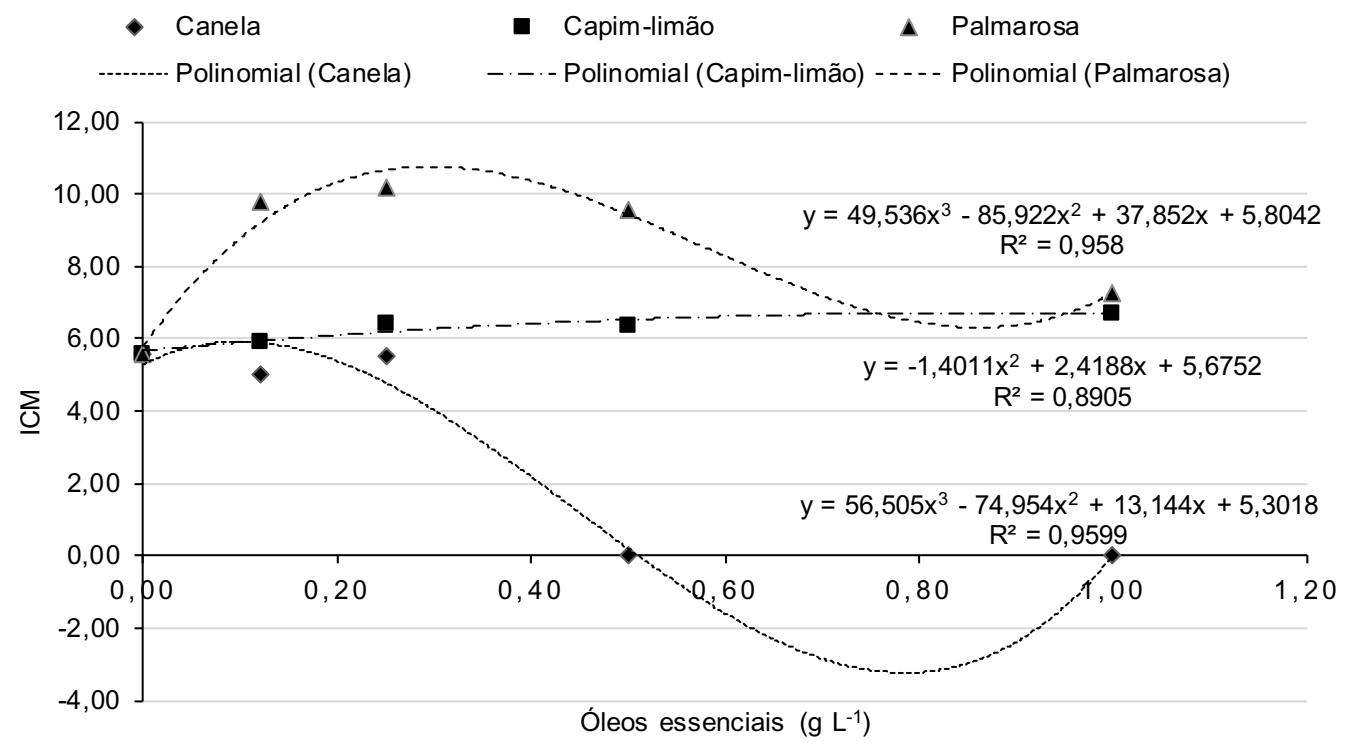

Figura 1. Índice de crescimento micelial (ICM) de Penicillium digitatum submetido a diferentes tratamentos com óleos essenciais por contato $\left(0,0 ; 0,12 ; 0,25 ; 0,50 ; 1,0 \mathrm{~g} \mathrm{~L}^{-1}\right)$, in vitro, a $25^{\circ} \mathrm{C}$ por 7 dias. Média de sete repetições.

Tabela 1. Diâmetro da colônia ( $\mathrm{mm}$ ) e índice de crescimento micelial (ICM) de Penicillium digitatum submetido a tratamentos com óleos essenciais por volatilização, in vitro, a $25^{\circ} \mathrm{C}$ por 4 dias.

\begin{tabular}{lccccc}
\hline \multirow{2}{*}{ Tratamentos } & \multicolumn{5}{c}{ Dias } \\
\cline { 2 - 5 } & $\mathbf{1}$ & $\mathbf{2}$ & $\mathbf{3}$ & $\mathbf{4}$ & ICM \\
\hline Testemunha & $7,72 \mathrm{a}^{*}$ & $18,51 \mathrm{a}$ & $32,54 \mathrm{a}$ & $41,04 \mathrm{a}$ & $8,33 \mathrm{a}$ \\
Canela & $5,46 \mathrm{c}$ & $14,74 \mathrm{c}$ & $25,95 \mathrm{c}$ & $33,58 \mathrm{~b}$ & $7,03 \mathrm{~b}$ \\
Capim-limão & $6,12 \mathrm{bc}$ & $16,38 \mathrm{~b}$ & $30,05 \mathrm{~b}$ & $38,64 \mathrm{a}$ & $8,13 \mathrm{a}$ \\
Palmarosa & $6,45 \mathrm{~b}$ & $17,72 \mathrm{ab}$ & $30,81 \mathrm{ab}$ & $40,60 \mathrm{a}$ & $8,53 \mathrm{a}$ \\
\hline CV\% & 10,38 & 6,05 & 4,46 & 4,84 & 5,30 \\
\hline
\end{tabular}

*Média de sete repetições. Médias seguidas de mesma letra na coluna não diferem significativamente entre si (Tukey, $p \leq 0,05$ ).
A inibição do desenvolvimento do bolor verde (AACPD) em laranjas inoculadas (efeito curativo) foi mais efetiva pelo tratamento com óleo de canela $\left(0,5 \mathrm{~g} \mathrm{~L}^{-1}\right)$, mesmo não diferindo significativamente da testemunha. O óleo de capim-limão e de palmarosa não mostraram ação antifúngica nas doses testadas e estimularam o desenvolvimento das lesões nos frutos (Figura 2). Quanto ao efeito protetivo dos óleos contra o bolor verde (Figura 3), verificou-se que o capim-limão $(0,5$ $\mathrm{g} \mathrm{L}^{-1}$ ) proporcionou uma redução de $53 \%$ no valor de AACPD em relação à testemunha.

Diante dos resultados mais promissores observados com o óleo de canela, realizou-se um teste screening com este óleo e um blend com o capim-limão. Verificou-se que canela a $0,12 \mathrm{~g} \mathrm{~L}^{-1}$ foi o tratamento que mais inibiu o desenvolvimento das lesões do bolor verde nas laranjas nos dois primeiros dias. O blend de canela e capim-limão não apresentou

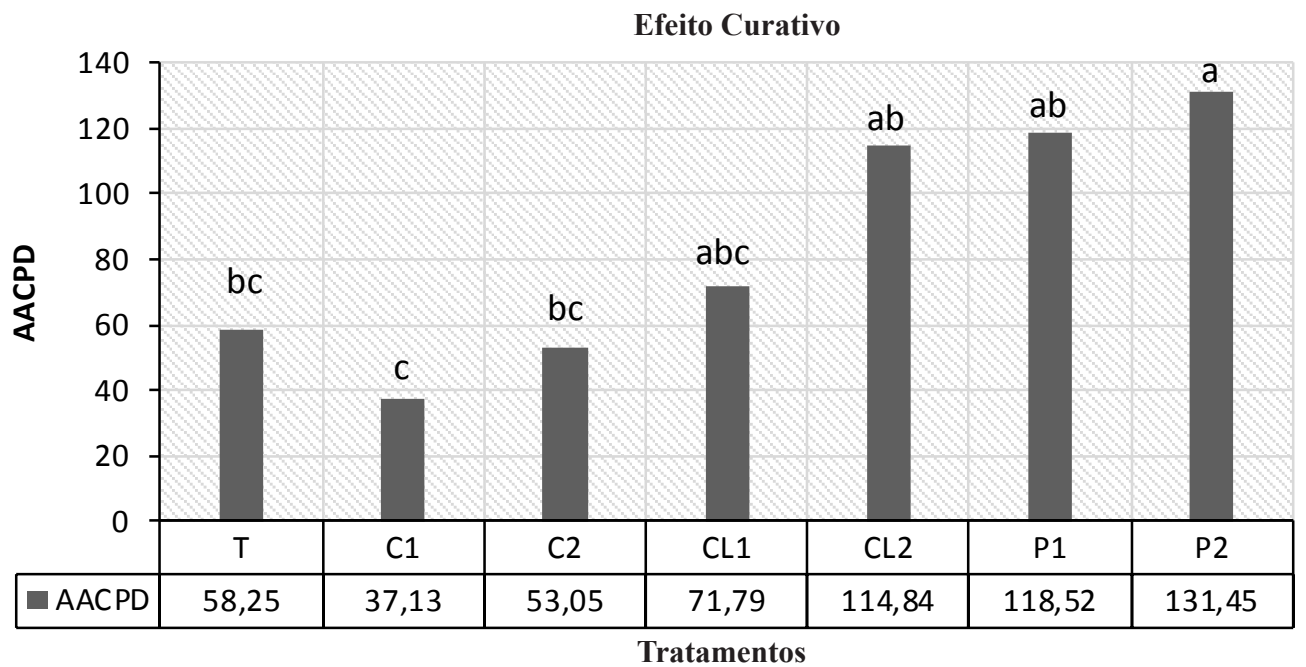

Figura 2. Área abaixo da curva de progresso da doença (AACPD) para valores de diâmetro das lesões (mm) de bolor verde em laranjas inoculadas

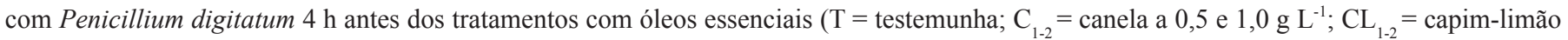
a 0,5 e $1,0 \mathrm{~g} \mathrm{~L}^{-1} ; \mathrm{P}_{1-2}=$ palmarosa a 0,5 e $1,0 \mathrm{~g} \mathrm{~L}^{-1}$ ), armazenadas a $25^{\circ} \mathrm{C} / 80 \% \mathrm{UR}$ por 4 dias. Média de 10 repetições. Letras distintas na coluna representam diferença significativa entre os tratamentos (Tukey, $\mathrm{p} \leq 0,05$ ). Para efeito de análise estatística, os dados foram transformados em raiz de $\sqrt{x+0,5} \cdot \mathrm{CV}=38,98 \%$. 


\section{Efeito Protetivo}

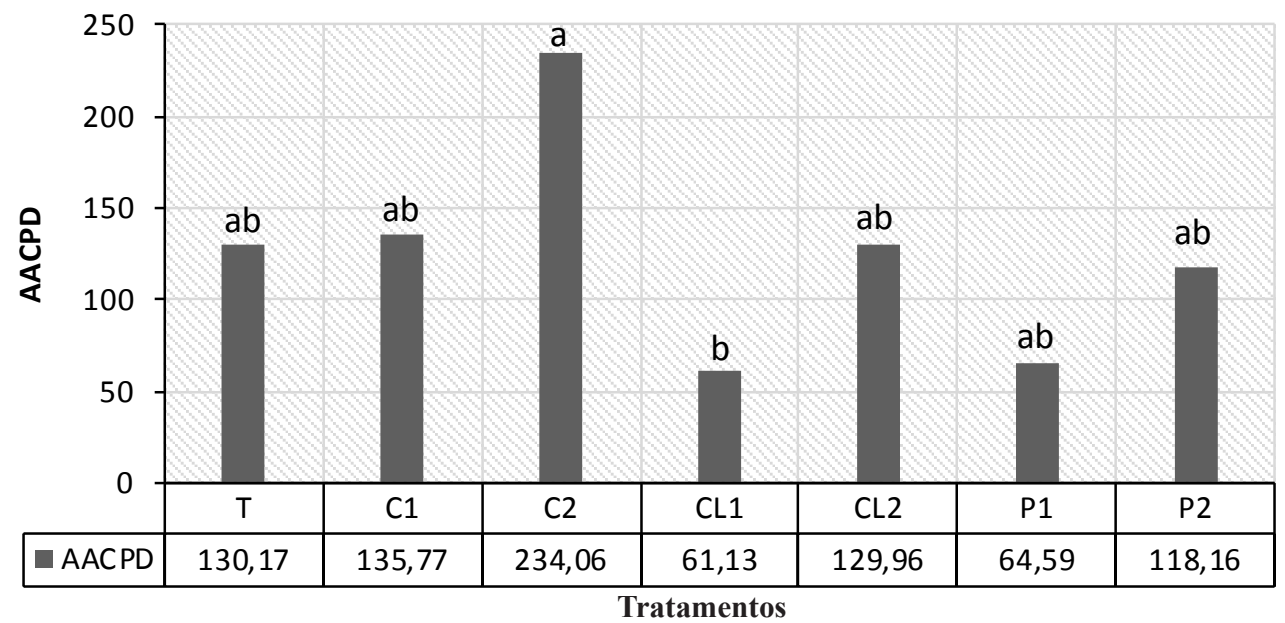

Figura 3. Área abaixo da curva de progresso da doença (AACPD) para valores de diâmetro das lesões (mm) de bolor verde em laranjas inoculadas

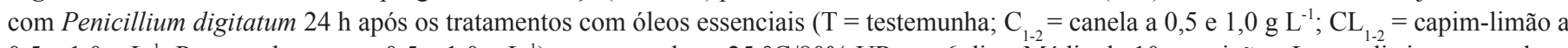
0,5 e $1,0 \mathrm{~g} \mathrm{~L}^{-1} ; \mathrm{P}_{1-2}=$ palmarosa a 0,5 e $1,0 \mathrm{~g} \mathrm{~L}^{-1}$ ), armazenadas a $25{ }^{\circ} \mathrm{C} / 80 \%$ UR por 6 dias. Média de 10 repetições. Letras distintas na coluna representam diferença significativa entre os tratamentos (Tukey, $\mathrm{p} \leq 0,05$ ). Para efeito de análise estatística, os dados foram transformados em raiz de $\sqrt{x+0,5} \cdot \mathrm{CV}=80,81 \%$.

controle da podridão nas laranjas (Figura 4).

No ensaio para comparar o uso do óleo essencial com outros métodos de controle (Figura 5), constatou-se que a combinação de aspersão do óleo de canela $\left(0,12 \mathrm{~g} \mathrm{~L}^{-1}\right)$ após o tratamento térmico dos frutos reduziu significativamente o desenvolvimento do bolor verde (AACPD), quando armazenados sob refrigeração $\left(10^{\circ} \mathrm{C}\right)$, entretanto, o tratamento com o fungicida imazalil proporcionou um controle mais efetivo (93\%) durante o armazenamento prolongado.

Neste trabalho, in vitro, observou-se que o óleo de canela foi o mais fungitóxico para $P$. digitatum, inibindo totalmente o ICM em concentrações superiores a $0,5 \mathrm{~g} \mathrm{~L}^{-1}$ por contato e, reduzindo significativamente pelos seus constituintes voláteis $\left(\approx 170 \mathrm{uL} \mathrm{L}^{-1}\right)$.

Vários trabalhos também encontraram resultados que corroboram com este. Lorenzetti et al. (13), ao analisarem o efeito de vários óleos essenciais sobre o desenvolvimento de $B$. cinerea, in vitro, constataram maior eficiência do óleo de canela, seguido de capimlimão e palmarosa por contato, enquanto, os voláteis de capim-limão e canela foram os que mais inibiram o ICM. Combrinck et al. (3) evidenciaram o efeito antimicrobiano do óleo de canela e de seu composto majoritário (eugenol) sobre $P$. digitatum. Mahmoud (14) também constatou atividade fungitóxica de extrato de canela sobre $P$. digitatum e Aspergillus niger van Tiegh.

Os componentes majoritários eugenol e citral, respectivamente, de óleo de canela e capim-limão apresentam alta atividade antimicrobiana $(2,3)$. A ação antimicrobiana do eugenol está relacionada com a capacidade de permeabilizar a membrana celular e interagir com as proteínas, inibindo a atividade das enzimas: ATPase, histidina decarboxylase, amilase e protease, ou seja, prejudica a biossíntese de

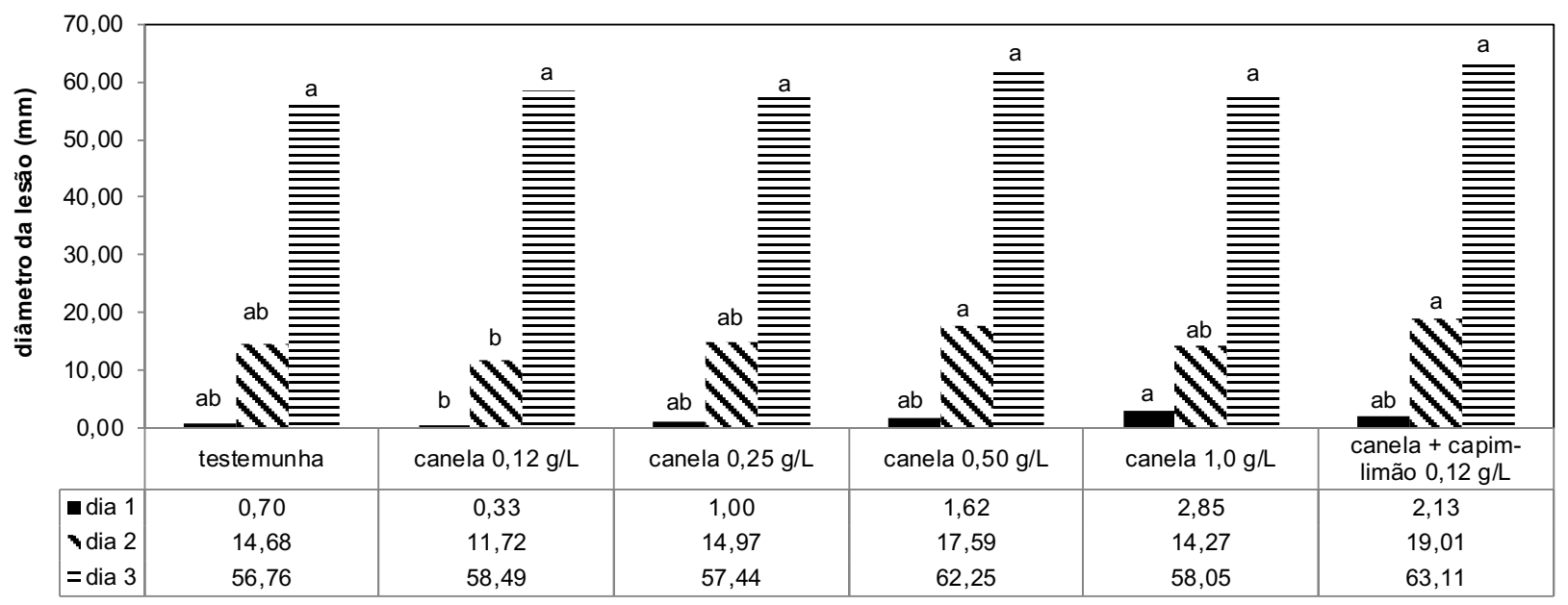

Tratamentos

Figura 4. Diâmetro das lesões $(\mathrm{mm})$ de bolor verde em laranjas inoculadas $4 \mathrm{~h}$ antes dos tratamentos com diferentes concentrações de óleo essencial de canela e armazenadas a $25^{\circ} \mathrm{C} / 80 \%$ UR por 3 dias. Média de 10 repetições. Letras distintas na coluna representam diferença significativa entre os tratamentos (Tukey, $\mathrm{p} \leq 0,05$ ). Coeficiente de variação (CV) para 1, 2 e 3 dias, respectivamente, 120,73; 24,97 e 10,62\%. 


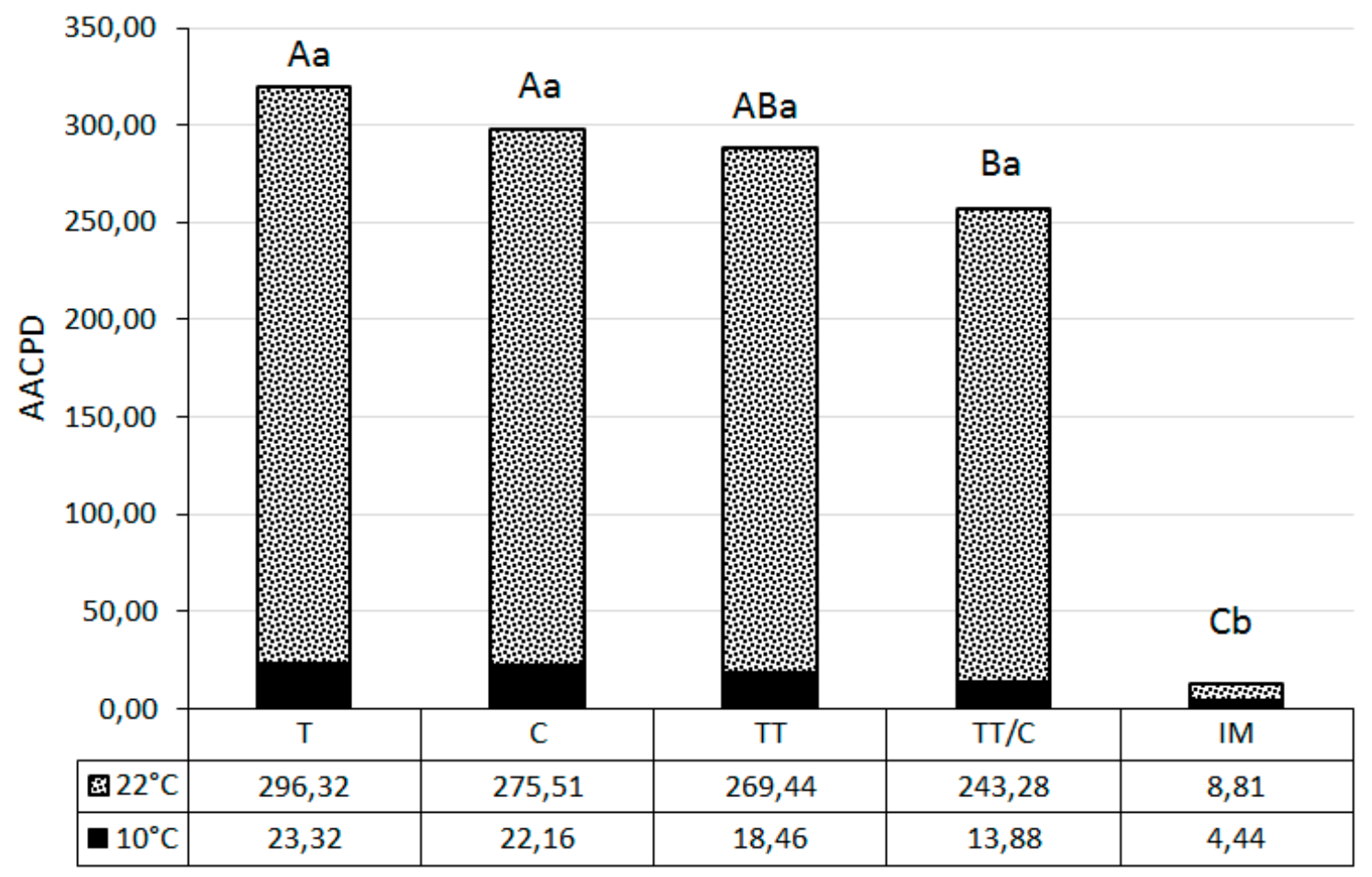

Tratamentos

Figura 5. Área abaixo da curva de progresso da doença (AACPD) para valores de diâmetro das lesões (mm) de bolor verde em laranjas 'Pera' inoculadas com Penicillium digitatum $4 \mathrm{~h}$ antes dos tratamentos: $\mathrm{T}=$ testemunha (água); $\mathrm{C}=$ canela $\left(0,12 \mathrm{~g} \mathrm{~L}^{-1}\right)$; $\mathrm{TT}=$ tratamento térmico spray $\left(60{ }^{\circ} \mathrm{C} / 20 \mathrm{~s}\right) ; \mathrm{IM}=$ imazalil $\left(1000 \mathrm{mg} \mathrm{L}^{-1}\right)$; com armazenamento refrigerado a $10{ }^{\circ} \mathrm{C} / 85 \%$ UR por 6 dias e mais 3 dias sob condições ambiente $\left(22^{\circ} \mathrm{C} / 70 \% \mathrm{UR}\right)$. Média de seis repetições com seis frutos por parcela. Letras maísculas e minúsculas na coluna representam, respectivamente, a comparação estatística do armazenamento a $10^{\circ} \mathrm{C}$ e $22^{\circ} \mathrm{C}$ (Tukey, $\mathrm{p} \leq 0,05$ ).

proteínas e enzimas, causa falhas nos processos biossintéticos e de geração energética celular (12).

A grande maioria dos estudos sobre a atividade antimicrobiana dos óleos essenciais são realizados in vitro, entretanto, nem sempre a concentração eficaz in vitro é a melhor quando aplicada no produto vegetal (in vivo), podendo acarretar fitotoxicidade, prejudicando a qualidade e até favorecendo o desenvolvimento de podridões $(1,2,18)$.

Avaliando o efeito curativo e protetivo dos óleos, pôde-se notar que o óleo de canela foi mais efetivo como curativo e, capim-limão como protetivo. Além disso, a dose $0,5 \mathrm{~g} \mathrm{~L}^{-1}$ mostrou sempre os melhores resultados na redução do bolor verde em laranjas quando comparada a $1,0 \mathrm{~g} \mathrm{~L}^{-1}$, provavelmente, devido à fitotoxicidade (Figuras 2 e 3). Entretanto, a dose de $0,12 \mathrm{~g} \mathrm{~L}^{-1}$ do óleo de canela foi a única que mostrou efeito positivo no teste screening e frente ao blend de canela mais capimlimão (Figura 4). Ao comparar com outros tratamentos, o efeito curativo do óleo de canela $\left(0,12 \mathrm{~g} \mathrm{~L}^{-1}\right)$ foi significativo quando combinado com o tratamento térmico $\left(60{ }^{\circ} \mathrm{C} / 20 \mathrm{~s}\right)$ e sob armazenamento refrigerado $\left(10^{\circ} \mathrm{C}\right)$, porém, quando os frutos foram transferidos para condições ambiente, observou-se o efeito fungistático destes tratamentos pelo rápido desenvolvimento das lesões, diferentemente do efeito fungicida do imazalil a $1000 \mathrm{mg} \mathrm{L}^{-1}$ (Figura 5).

Soylu et al. (19) verificaram efeito fungitóxico de óleo de orégano, in vitro e in vivo, no controle de $B$. cinerea em tomate produzido em casa-de-vegetação, com maior porcentagem de controle curativo do que protetivo. Sobre este aspecto, os autores referem que os diferentes constituintes voláteis e majoritários dos óleos podem ser responsáveis pelo melhor efeito curativo, por serem mais prontamente absorvidos pelo micélio e estruturas de reprodução do fungo. O mecanismo de ação do efeito protetivo precisa ser melhor investigado.

A qualidade dos óleos essenciais depende de vários parâmetros como gravidade específica, índice de refração, solubilidade em diferentes solventes orgânicos, valor de saponificação, conteúdo de fenólicos, grau de volatilidade, etc. Além disso, alguns fatores influenciam a composição química dos óleos como local de produção, clima, período de colheita e técnicas de destilação, que podem ocasionar variabilidade nas propriedades antimicrobianas (21).

As interações entre os constituintes químicos presentes nos óleos, quando combinados, podem ser definidas por uma ou mais moléculas da constituição química e podem acarretar efeitos antagônicos, sinérgicos ou aditivos. Um exemplo de ação antimicrobiana sinérgica foi relatado pela combinação ternária de carvacrol, timol e eugenol; por outro lado, uma ação antagônica foi a combinação de carvacrol com óleo de orégano (12). A interação do óleo de canela com capim-limão, ou com outros óleos de constituintes químicos diferentes necessitam de mais pesquisas, quanto ao $\mathrm{FIC}_{\text {index }}$ (índice da concentração fracionada inibitória), que é obtido pelo estudo da MIC (mínima concentração inibitória) para componentes individuais dos óleos.

Mais trabalhos relatam resultados positivos no uso de óleos essenciais e extratos de plantas para controle de $P$. digitatum em frutos cítricos, como: tomilho (6); extrato de neem, pong-pong e pimenta (1); timol e carvacrol (15).

A combinação de métodos também pode ser sinérgica, como a aplicação de biofungicidas ou produtos alternativos (óleos essenciais), após o tratamento térmico, pela somatória de efeitos sobre o patógeno e/ou por elicitar mecanismos de resistência nos frutos $(10,16)$.

Resultados comparativos entre o uso de óleos essenciais e 
fungicidas convencionais também são encontrados na literatura. Tripathi et al. (21) verificaram a MIC dos óleos essenciais de manjericão santo, folhas de pêssego e gengibre, respectivamente, 200, 100 e 100 $\mathrm{mg} \mathrm{L}^{-1}$ contra $B$. cinerea, em contrapartida, as MICs de fungicidas sintéticos foram mais altas como benomyl $\left(3000 \mathrm{mg} \mathrm{L}^{-1}\right)$ e ziram $(5000$ $\left.\mathrm{mg} \mathrm{L}^{-1}\right)$. Estes óleos, aplicados por volatilização em uvas inoculadas, apresentaram significativa atividade fungitóxica e extensão da vida útil no armazenamento, sem qualquer sintoma de fitotoxicidade. Anaruma et al. (1) também constataram significativa atividade fungitóxica de capim-limão $\left(0,12 \mathrm{~g} \mathrm{~L}^{-1}\right)$ contra $C$. gloeosporioides em maracujá amarelo, com resultado semelhante ao fungicida prochloraz. Doses superiores do óleo causaram fitotoxicidade.

A identificação de óleos essenciais, com propriedades antimicrobianas para determinados patossistemas, tem se mostrado como uma potencial ferramenta para formulação de produtos alternativos e que podem ser combinados com outros métodos, como tratamento térmico, refrigeração, atmosfera modificada, a serem aplicados no manejo da agricultura convencional e orgânica.

\section{AGRADECIMENTO}

Ao $\mathrm{CNPq}$ pela bolsa PIBIC; à FAPESP pelo apoio financeiro (processo 2011/23432-8).

\section{REFERÊNCIAS}

1. Al-Samarrai, G. F.; Singh, H.; Syarhabil, M. Extracts some plants on controlling green mold of orange and on postharvest quality parameters. World Applied Sciences Journal, Faisalabad, v. 22, n.4, p. 564-570, 2013.

2. Anaruma, N.D.; Schmidt, F.L.; Duarte, M.C.T.; Figueira, G.M.; Delarmelina, C.; Benato, E.A.; Sartoratto, A. Control of Colletotrichum gloeosporioides (Penz.) Sacc. in yellow passion fruit using Cymbopogon citratus essential oil. Brazilian Journal of Microbiology, São Paulo, v.41, p.66-73, 2010.

3. Combrinck, S.; Regnier, T.; Kamatou, G.P.P. In vitro activity of eighteen essential oils and some major components against common postharvest fungal pathogens of fruit. Industrial Crops and Products, Amsterdam, v.33, p.344-349, 2011.

4. De Capdeville, G.; Wilson, C.L.; Beer, S.V.; Aist, J.R. Alternative disease control agentes induce resistance to blue mold in harvest 'Red Delicious' apple fruit. Phytopathology, St. Paul, v.92, p.900-908, 2002.

5. Demyttenaere, J.C.R.; Herrera, M.D.C.; Kimpe, N. De. Biotransformation of geraniol, nerol and citral by sporulated surface cultures of Aspergillus niger and Penicillium sp. Phytochemistry, New York, v. 55, p.363-373, 2000.

6. Fatemi, S.; Jafarpour, M.; Eghbalsaied, S.; Rezapour, A.; Borji, H. Effect of essential oils of Thymus vulgaris and Mentha piperita on the control of green mould and postharvest quality of Citrus Sinensis cv. Valencia. African Journal of Biotechnology, Nairobi, v.10, n.6, p.14932-14936, 2011.

7. Fischer, I.H.; Lourenço, S.A.; Amorim, L. Doenças pós-colheita em citros e caracterização da população fúngica ambiental no mercado atacadista de São
Paulo. Tropical Plant Pathology, Brasília, vol. 33, n.3, p.219-226, 2008

8. Fisher, K. \& Phillips, C. Potential antimicrobial uses of essential oils in food: is citrus the answer? Trends in Food Science \& Technology, Cambridge, v.19, p.156-164, 2008.

9. Forner, C.; Bettiol, W.; Nascimento, L.M.; Terao, D. Controle em pós-colheita de Penicillium digitatum em laranja 'Pera' com microrganismos e tratamento térmico. Revista Brasileira de Fruticultura, Jaboticabal, v.35, n.1, p.023-031, 2013.

10. Franco, D.A.S. \& Bettiol, W. Efeito de produtos alternativos para o controle do bolor verde (Penicillium digitatum) em pós-colheita de citros. Revista Brasileira de Fruticultura, Jaboticabal, v.24, n.2, p.569-572, 2002.

11. Guimarães, L.G.L.; Cardoso, M.G.; Souza, P.E.; Andrade, J.; Vieira, S.A. Atividade antioxidante e fungitóxica do óleo essencial de capim-limão e do citral. Revista Ciência Agronômica, Fortaleza, v.42, n.2, p.464-472, 2011.

12. Hyldgaard, M.; Mygind, T; Meyer, R. Essential oils infood preservation: modeofaction, synergies, and interactions with food matrix components. Frontiers in Microbiology, Lausanne, v.3, p.1-12, 2012.

13. Lorenzetti, E.R.; Monteiro, F.P.; Souza, R.J.; Scalice, H.K.; Diogo Jr., R.; Pires, M.S.O. Bioatividade de óleos essenciais no controle de Botrytis cinerea isolado de morangueiro. Revista Brasileira de Plantas Medicinais, Botucatu, v.13, especial, p.619-627, 2011.

14. Mahmoud, S.N. Antifungal activity of Cinnamomum zeylanicum and Eucalyptus microtheca crude extracts against food spoilage fungi. Euphrates Journal of Agriculture Science, Al-kasim, v.4, n.3, p.26-39, 2012.

15. Pérez-Alfonso, C.O.; Martínez-Romero, D.; Zapata, P.J.; Serrano, M.; Valero, D.; Castillo, S. The effects of essential oils carvacrol and thymol on growth of Penicillium digitatum and P. italicum involved in lemon decay. International Journal of Food Microbiology, Amsterdam, v.158, p.101-106, 2012.

16. Porat, R.; Daus, A.; Weiss, B.; Cohen, L.; Fallik, E.; Droby, S. Reduction of postharvest decay in organic citrus fruit by a short hot water brushing treatment. Postharvest Biology and Technology, Amsterdam, v.18, p.151-157, 2000.

17. Rozwalka, L.C.; Lima, M.L.R.Z.C.; De Mio, L.L.M.; Nakashima, T. Extratos, decoctos e óleos essenciais de plantas medicinais e aromáticas na inibição de Glomerella cingulata e Colletotrichum gloeosporioides de frutos de goiaba. Ciência Rural, Santa Maria, v.38, n.2, p.301-307, 2008.

18. Shao, X.; Cao, B.; Xu, F.; Xie, S.; Yu, D.; Wang, H. Effect of postharvest application of chitosan combined with clove oil against citrus green mold. Postharvest Biology and Technology, Amsterdam, v.99, p.37-43, 2015.

19. Soylu, E.M; Kurt, SS.; Soylu, S. In vitro and in vivo antifungal activities of the essential oils of various plants against tomato grey mould disease agent Botrytis cinerea. International Journal of Food Microbiology, Amsterdam, v.143, n. 183-189, 2010.

20. Tripathi, P. \& Dybey, N.K. Exploitation of natural products as an alternative strategy to control postharvest fungal rotting of fruit and vegetables. Postharvest Biology and Technology, Amsterdam, v.32, p.235-245, 2004

21. Tripathi, P.; Dybey, N.K.; Shukla, A.K. Use of some essential oils as post-harvest botanical fungicides in the management of grey mould of grapes caused by Botrytis cinerea. World Journal Microbiology and Biotechnology, Oxford, v.24, p.39-46, 2008.

22. Whiteside, J.O.; Garnsey, S.M.; Timmer, W. (ed.). Compendium of citrus diseases. St. Paul: APS, 1989. 80p.

23. Yahyazadeh, M.; Omidbaigi, R.; Zare, R.; Taheri, H. Effect of some essential oils on mycelial growth of Penicillium digitatum Sacc. World Journal of Microbiology and Biotechnology, Oxford, v.24, p.1445-1450, 2008. 